\title{
BMJ Open Reducing Medical Admissions into Hospital through Optimising Medicines (REMAIN HOME) Study: protocol for a stepped-wedge, cluster-randomised trial
}

\author{
Holly Foot, ${ }^{1}$ Christopher Freeman, ${ }^{1}$ Karla Hemming, ${ }^{2}$ lan Scott, ${ }^{3,4}$ \\ Ian D Coombes, ${ }^{1,5}$ Ian D Williams, ${ }^{6,7}$ Luke Connelly, ${ }^{8,9}$ Jennifer A Whitty, ${ }^{1,10}$ \\ Nancy Sturman, ${ }^{1}$ Sue Kirsa, ${ }^{11,12}$ Caroline Nicholson, ${ }^{13}$ Grant Russell, ${ }^{14}$ \\ Carl Kirkpatrick, ${ }^{12}$ Neil Cottrell ${ }^{1}$
}

To cite: Foot $\mathrm{H}$, Freeman $\mathrm{C}$, Hemming $\mathrm{K}$, et al. Reducing Medical Admissions into Hospital through Optimising Medicines (REMAIN HOME) Study: protocol for a stepped-wedge, clusterrandomised trial. BMJ Open 2017;7:e015301. doi:10.1136/ bmjopen-2016-015301

- Prepublication history and additional material are available. To view these files please visit the journal online (http://dx.doi org/10.1136/bmjopen-2016015301)

$\mathrm{HF}$ and CF contributed equally.

Received 24 November 2016 Revised 9 February 2017 Accepted 9 March 2017

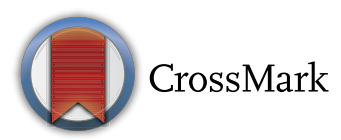

For numbered affiliations see end of article.

Correspondence to Dr Christopher Freeman; c.freeman4@uq.edu.au

\section{ABSTRACT}

Introduction A model of general practitioner (GP) and pharmacist collaboration in primary care may be an effective strategy to reduce medication-related problems and provide better support to patients after discharge. The aim of this study is to investigate whether a model of structured pharmacist and GP care reduces hospital readmissions in high-risk patients.

Methods and analysis This protocol details a stepped-wedge, cluster-randomised trial that will recruit participants over 9 months with a 12-month follow-up. There will be 14 clusters each representing a different general practice medical centre. A total of 2240 participants will be recruited from hospital who attend an enrolled medical centre, take five or more long-term medicines or whose reason for admission was related to heart failure or chronic obstructive pulmonary disease. The intervention is a multifaceted service, involving a pharmacist integrated into a medical centre to assist patients after hospitalisation. Participants will meet with the practice pharmacist and their GP after discharge to review and reconcile their medicines and discuss changes made in hospital. The pharmacist will follow-up with the participant and liaise with other health professionals involved in the participant's care. The control will be usual care, which usually involves a patient self-organising a visit to their GP after hospital discharge. The primary outcome is the rate of unplanned, all-cause hospital readmissions over 12 months, which will be analysed using a mixed effects Poisson regression model with a random effect for cluster and a fixed effect to account for any temporal trend. A cost analysis will be undertaken to compare the healthcare costs associated with the intervention to those of usual care.

Ethics and dissemination The study has received ethical approval (HREC/16/QRBW/410). The study findings will be disseminated through peer-reviewed publications, conferences and reports to key stakeholders.

Trial registration number ACTRN12616001627448

\section{INTRODUCTION}

The transition of patients with chronic and complex conditions from hospital to the
Strengths and limitations of this study

- A model of GP and pharmacist collaboration provides an opportunity to improve health outcomes in patients discharged from hospital.

- The stepped-wedge study design is robust and pragmatic, enabling the intervention to be offered to every medical centre involved by the end of the study period.

- Multi-skilled research team involving clinicians, health services researchers, a biostatistician and health economists.

- Strong stakeholder participation contributing to the protocol design.

- Blinding of the intervention is not possible to participants and staff at the medical centre.

community setting is a critical time that is associated with medication misadventure and re-hospitalisation. ${ }^{1} \mathrm{Up}$ to $50 \%$ of patients discharged from medical wards have an unplanned readmission within 1 year. $^{2-4}$ Rates are higher in the elderly and those on multiple medications. ${ }^{5-7}$

Medication-related problems are one of the most common reasons for readmissions to hospital and include adverse drug events, suboptimal therapy and poor medication adherence. ${ }^{48}$ Patients often leave hospital with complex care plans and substantial changes to their medication regimens. ${ }^{9}$ In Australia, these changes are usually communicated to the patient's general practitioner (GP), community pharmacy and other health professionals involved in the patient's care via a discharge summary. Discharge summaries can take time to reach primary care providers and often lack key information, such as medication changes, pathology tests that were performed or are pending and agreed treatment plans. ${ }^{10}$ This 
can lead to medication discrepancies, such as continuing discontinued medication, omitting prescribed medication and not implementing dose changes. ${ }^{11}$ Patients who have a medication discrepancy are twice as likely to be readmitted to hospital within 30 days. ${ }^{12}$

There have been a number of interventions targeted towards optimising medicine use and improving the timeliness of information leaving hospitals to minimise medication discrepancies and reduce hospital readmissions. ${ }^{13-16}$ A common intervention used to reduce hospital readmissions is medication reconciliation. The literature exploring the efficacy of medication reconciliation at hospital discharge to reduce readmissions is mixed. A recent meta-analysis investigated the effect of pharmacist-led medication reconciliation at hospital transition. ${ }^{16}$ Most of the included studies matched a patient's preadmission medication to their discharge medication and reconciled any differences. The meta-analysis showed there was a $19 \%$ reduction in readmissions for those receiving pharmacist-led medication reconciliation compared with usual care. ${ }^{16}$ Other outcomes included a reduction in emergency department (ED) visits $(28 \%)$ and adverse drug event-related readmissions (67\%). These reductions would be anticipated to be associated with substantial cost savings. The findings differ to an earlier systematic review that reported hospital staff-led medication reconciliation had minimal effect on readmissions. ${ }^{17}$ However, many of the studies included in the earlier systematic review did not recruit high-risk patients, which may be one reason why certain interventions are not successful. Previous hospitalisations or ED visits, taking multiple long-term medicines and being admitted for heart failure or chronic obstructive pulmonary disease (COPD) are common risk factors for readmission. ${ }^{36718}$ Interventions targeting participants with these risk factors appear more effective at reducing readmissions. ${ }^{17} 19$

Another approach to reducing readmissions has been to conduct a medication review immediately after discharge ${ }^{20-22}$ Pharmacists working within a primary care clinic undertaking medication review, with a focus on medication reconciliation and patient education, showed a reduction in hospital readmissions in two studies. ${ }^{20} 21$ Tedesco $e t a l^{22}$ were limited by the study design and small sample size, although they observed a trend $(\mathrm{p}=0.27)$ towards decreased readmissions in the intervention group compared with control. The authors noted that there were fewer readmissions in those who interacted with the pharmacist face to face versus by telephone $(\mathrm{p}=0.05)$.

Overall, common characteristics among effective interventions include allowing for direct communication between the pharmacist and the prescriber, ${ }^{23}$ involving postdischarge care ${ }^{20}$ and targeting high-risk patients. ${ }^{171924}$ A model of GP and pharmacist collaboration assisting patients during transitions of care may be a clinically relevant and cost-effective strategy to reduce unplanned readmissions.

A practice pharmacist is 'a pharmacist who delivers professional services from or within a general practice medical centre with a coordinated, collaborative and integrated approach with an overall goal to improve the quality use of medicines of the practice population'. ${ }^{25}$ Despite widespread support, the Australian setting is in its infancy in exploring this model of practice that is restricted by the absence of a dedicated and sustainable remuneration model. ${ }^{26}$ The vast majority of Australian pharmacists working in primary care do so in a community pharmacy with one study reporting only 26 pharmacists working within or from a general practice in $2013 .^{25}$ Although performing comprehensive medication reviews is a core activity of practice pharmacists, they are also involved in a range of clinician-level and practice-level activities. These include providing drug information and education as well as clinical prescribing review with prescriber feedback. ${ }^{26}$ Being co-located within the patient's medical home allows for greater communication and collaboration with primary healthcare providers and access to patient's medical records. ${ }^{27} 28$ This allows quick identification and resolution of medication-related problems with the prescribing GP, linking with community pharmacies to enhance continuity of care and liaising with hospitals when patients are discharged. Practice pharmacists integrated into the general practice medical team have shown favourable outcomes, with improvements in clinical outcomes, identification and reduction in medication-related problems and improved medication adherence. ${ }^{27} \mathrm{~A}$ study in the Netherlands is currently exploring whether integrating non-dispensing pharmacists into the primary care team can reduce medication-related hospital admissions in patients considered at risk of adverse drug events. $^{29}$

A model of GP and pharmacist collaboration provides an opportunity to improve information transfer between hospitals and primary care, reduce medication-related problems and discrepancies, and provide better support to patients after discharge to reduce unplanned hospital readmissions.

The aim of this study is to investigate whether a model of structured pharmacist and GP care reduces unplanned hospital readmissions in high-risk patients.

The primary objective is to investigate whether integrating a pharmacist into the general practice team will reduce unplanned hospital readmissions at 12 months.

Secondary objectives are to:

1. Investigate whether integrating a pharmacist into the general practice team will:

a. reduce unplanned hospital readmissions at 30 days, 3 and 6 months;

b. reduce ED presentations at 30 days, 3, 6 and 12 months;

c. improve the timeliness of receiving the hospital discharge treatment plan in primary care;

d. increase the number of participants visiting their GP within 1 week of discharge.

2. assess the cost savings associated with a model of GP-pharmacist collaboration of care in reducing hospital readmissions; 
3. describe the activities performed by practice pharmacists and GPs when transitioning a patient from hospital to primary care;

4. explore the views and experiences of the intervention from the perspective of the GP, practice pharmacists, community pharmacists and participants involved in the study.

\section{METHODS AND ANALYSIS}

This protocol was developed in accordance with the Standard Protocol Items: Recommendations for Interventional Trials (SPIRIT) 2013 Statement ${ }^{30}$ (see online supplementary SPIRIT 2013 checklist). Reference was also made to CONSORT statement extension for cluster randomised trials $^{31}$ and the recommended modifications for steppedwedge designs. ${ }^{32}$

\section{Study design and setting}

This is a stepped-wedge, cluster-randomised trial that will recruit participants over 9 months with a 12-month follow-up period. Participants will be recruited during their hospital admission from public and private hospitals across South East Queensland, Australia. The intervention will be located in 14 different medical centres. Cluster randomisation will be used to allow for randomisation to occur at the level of the cluster (medical centre) instead of the participant.

A stepped-wedge trial has a unidirectional cross-over design where all clusters (medical centres) begin in the control phase and cross over to the intervention at different time points. The design involves an initial period where no medical centres are exposed to the intervention (control phase). Then, at regular intervals, two medical centres will be randomised to cross over from control to the intervention. After 8 months, all medical centres will be exposed to the intervention. The length of time a medical centre receives the intervention may vary between 1 and 7 months (figure 1). A 1-month transition phase is included where the medical centre is not considered as being in control or intervention and does not contribute to analysis. This transition period allows for the time it takes to embed the intervention into a medical centre. ${ }^{32}$

This design was employed to: increase medical centre participation rates by offering the intervention to all 14 medical centres by the end of the study period; measure and adjust for possible underlying temporal trends (such as seasonal variation in admissions) and prevent potential direct/indirect educational effects of the intervention carrying over to the control phase (which precludes a traditional cross-over design).

\section{Study population}

Patients in hospital who are considered at risk of readmission and attending an enrolled medical centre will be invited to participate in the study.

\section{Eligibility criteria \\ Inclusion criteria}

1. have nominated a GP working in an enrolled medical centre in their hospital records;

2. prescribed $\geq 5$ long-term medicines on discharge OR primary discharge diagnosis of congestive heart failure OR exacerbation (either infective or noninfective) of COPD;

3. $\geq 18$ years of age.

\section{Exclusion criteria}

1. receiving active radiation therapy or chemotherapy for malignant conditions;

2. admission was for planned dialysis;

3 . in palliative care as reflected by the treatment regimen (eg, cessation of preventative medicines);

4. unable to attend a medication review and the follow-up within the time frame.

\section{Recruitment}

\section{Participants}

Patients in public and private hospitals located across the study geographical region who have met the eligibility criteria will be invited to participate in the study. Research

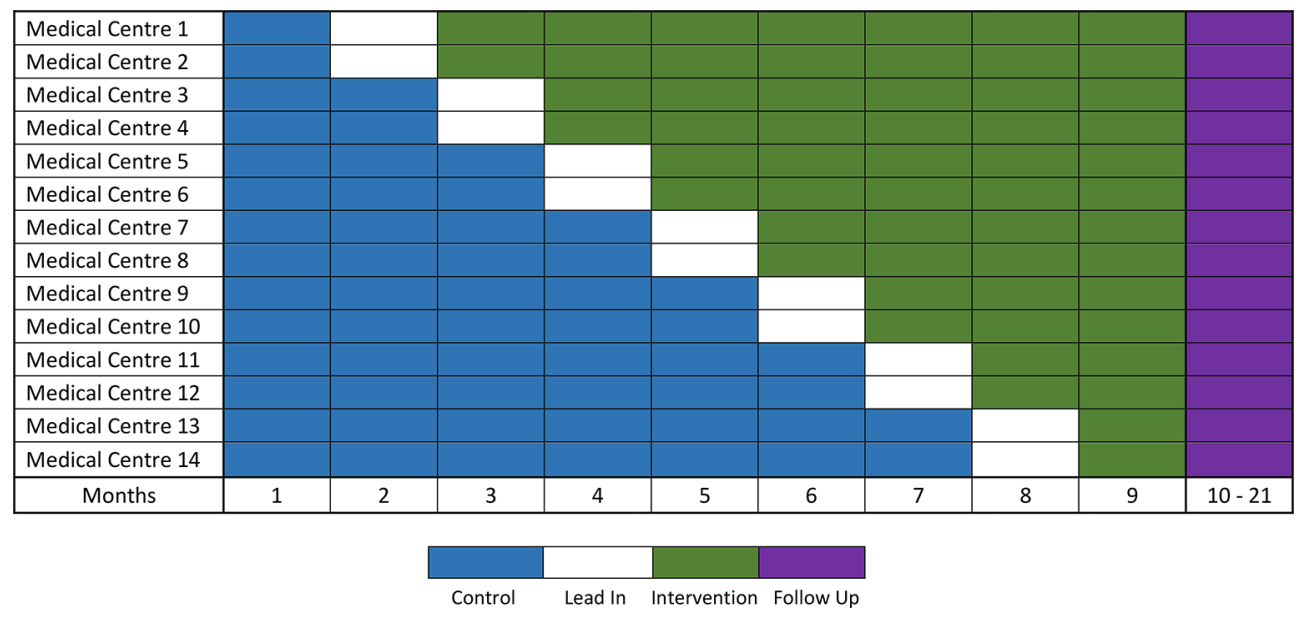

Figure 1 Schematic representation of the stepped-wedge study design. 
assistants will screen patients daily for eligibility and recruit those who give consent. Participants will comprise a convenience sample, as they will only be recruited when the research assistant is present.

\section{Medical centres}

To ensure we capture the required sample size, medical centres that are in a region that have a higher than average number of hospitalisations will be approached. To ensure the results are translatable, two of the 14 medical centres will be aboriginal community controlled health organisations that focus on improving the health of indigenous Australians. For logistical reasons, the aboriginal community controlled health organisations will be composed of groups of two to three small medical centres (treated as one single cluster for the purposes of randomisation).

\section{Inclusion criteria}

1. space to accommodate a co-located pharmacist in a private room;

2. anticipate capturing the required sample size $(20$ participants per month).

\section{Exclusion criteria}

1. Currently have a practice pharmacist integrated into the medical centre.

\section{Practice pharmacists}

Practice pharmacists will be recruited through an expression of interest advertisement distributed through national pharmacy associations. It will be essential that the pharmacist is registered to practice in Australia, has recent experience conducting medication reviews and working in a multidisciplinary team. All pharmacists will have professional registration and indemnity insurance. A particular pharmacist may work across multiple medical centres during the intervention. Medical centres randomised to have a short intervention phase may have a pharmacist that has had experience in a medical centre that has been randomised to have a longer intervention phase.

\section{Study procedure}

Following assessment of eligibility and participant consent, depending on which phase the cluster is in and time of recruitment, the participant will be allocated to the control or intervention. Figure 2 provides an overview of the study procedure.

\section{Control phase}

If the participant's medical centre is in the control phase, they will receive usual care. In Australia, usual care would mean the patient would consult their GP as per normal standards for that practice for a patient discharged from hospital. There will be no pharmacist in the medical centre during the control phase. Medication liaison in the form of a discharge medication record may be provided to patients on discharge from hospital and may be included in the hospital discharge summary to the GP.

\section{Lead in phase}

This phase will be used to assist the pharmacist integrating into the medical centre, prior to the intervention beginning. For greatest success, it will be imperative that the pharmacist establishes relationships with the GPs within the medical centre. To facilitate this, the practice pharmacist will be encouraged to meet with each GP, attend meetings and give a presentation to the medical staff on the role of the pharmacist in the study. The pharmacist may also visit the local community pharmacies to introduce themselves, explain the study and that they may be contacted by the practice pharmacist for referrals (home medicine reviews) and additional monitoring (blood pressure and others).

\section{Intervention phase}

If the participant's medical centre is in the intervention phase, the participant will receive the intervention.

The intervention is a multifaceted and collaborative service, involving a practice pharmacist integrated into a medical centre to assist patients in transitioning back into primary care after hospitalisation. The intervention is targeted at the level of the medical centre. A practice pharmacist will be integrated into each medical centre during the intervention phase for approximately 12 hours per week, spread across multiple days. There will be three components to the intervention that will apply to each participant in the intervention phase.

\section{Medication management consultation}

Participants will receive a face-to-face medication management consultation (approximately 45-60 min) with the practice pharmacist in a private room at the attended medical centre. In circumstances where the participant cannot attend their medical centre, the participant may elect to have a home visit by the practice pharmacist.

The initial medication management consultation will occur as soon as possible after discharge, ideally within five business days of discharge. During this time, the pharmacist will perform a comprehensive medication review to identify any medication-related problems, assess medication adherence, review the participant's medication discharge letter and discuss any changes made to medication during the hospital admission. The pharmacist will also review the participant's medical records at the practice and reconcile any differences as required. The pharmacist will discuss the intended treatment plan and any problems or concerns the participant may have regarding their medication and/or medical conditions. The pharmacist may also liaise with the participant's community pharmacy, hospital pharmacist and other prescribers to directly clarify any issues or anomalies with the participant's records and to communicate the changes made to the participant's medication regimen in hospital.

The practice pharmacist will also aim to enrol participants into a national online health record to allow better 


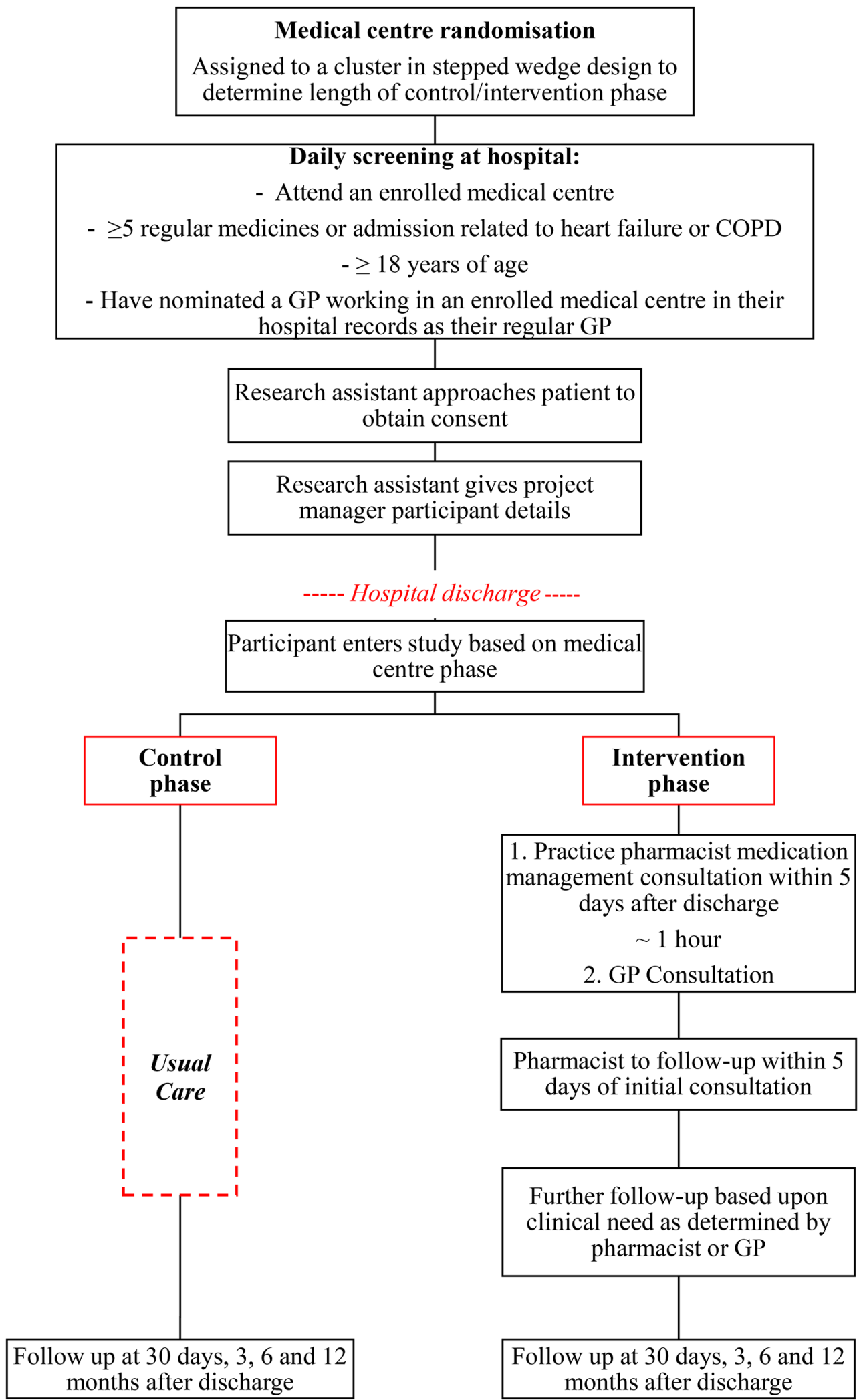

Figure 2 Diagram of REMAIN HOME patient recruitment, randomisation, intervention and control arms. COPD, chronic obstructive pulmonary disease; GP, general practitioner; REMAIN HOME, Reducing Medical Admissions into Hospital through Optimising Medicines. 
transfer of information between health professionals involved in the participant's care.

\section{Consultation with GP}

Directly after the consultation with the pharmacist, the participant will then have a consultation with their GP to consider any recommendations made by the pharmacist and discuss the recent admission to hospital and future management plans.

\section{Pharmacist follow-up}

The pharmacist will follow-up with the participant face to face or via the telephone within five business days of the initial consultation.

The pharmacist may also liaise with the participant's GP and other health professionals involved in the participant's care. This may include a referral to the participant's community pharmacy.

Additional follow-up with the participant will be determined by the pharmacist or GP based on clinical need for each participant.

\section{Outcome follow-up}

Participants will be followed up to 12 months from day of hospital discharge. This will be done through collection of routine data from the hospital and medical centre.

\section{Participant withdrawal}

For intention-to-treat purposes, we aim to collect routine data from all participants, regardless of whether they complete the study. If a participant decides to withdraw, we will request consent to collect their follow-up data.

\section{Medical centre withdrawal}

It is unlikely that medical centres will withdraw once they have agreed to take part. If a medical centre chooses not to fully engage with the intervention, the research team will request to collect follow-up data. This follow-up data collection will require minimal effort from practice staff. If a medical centre withdraws before commencement of recruitment, the next medical centre on the list will be contacted and invited to participate in the study.

\section{Outcomes}

The primary outcome is the rate of unplanned, all-cause hospital readmissions 12 months after discharge in the intervention group compared with control/usual care.

For the purposes of this study, an unplanned readmission is defined as any subsequent admission following the index hospital admission that is an emergency.

Secondary outcomes are:

1. the rate of unplanned, all-cause hospital readmissions at 30 days, 3 and 6 months after discharge in the intervention group compared with control/usual care;

2. the rate of ED presentations at 30 days, 3, 6 and 12 months after discharge in the intervention group compared with control/usual care;
3. number of hospital treatment plans received within five business days and 30 days after discharge in the intervention group compared with control/usual care;

4. number of participants reviewed by their GP within a week of discharge in the intervention group compared with control/usual care;

5. number of visits to the enrolled medical centre within 12 months after discharge in the intervention group compared with control/usual care;

6. costs to the healthcare system associated with the intervention (including the costs of providing the intervention, unplanned hospital readmissions, ED presentations and related GP visits) at 30 days, 3, 6 and 12 months after discharge in the intervention group compared with control/usual care;

7. description of views of the intervention from GPs, practice pharmacists, community pharmacists and participants involved in the study.

\section{Sample size}

Sample size was calculated for the primary outcome, the rate of unplanned, all-cause readmissions at 12 months from date of hospital discharge, using a method that takes into account the: intracluster correlation coefficient (ICC), expected baseline number of readmissions, effect of the intervention and power of the study. We followed methods described by others to determine the sample size needed, assuming a random effect for cluster (medical centre) and a fixed effect for each step to account for time. ${ }^{334}$ We have been conservative in our estimate of the ICC as we have limited a priori data to inform this value and also because the ICC is a process outcome, which usually have larger ICCs. ${ }^{35}$ Although the analysis will be a Poisson regression of rate of readmission, the study has been powered on a binary proportion outcome. This is because there is no data on which to estimate current rate of readmission and sample size methodology for steppedwedge studies does not currently exist for rate outcomes. This is a limitation of the sample size calculation, but the estimate of power will be conservative because the analysis will make full use of the number of readmissions.

Literature from Australian studies suggests that $45 \%$ of patients discharged from medical inpatient units and $61 \%$ from Geriatrics Evaluation and Management units have an unplanned readmission within 1 year. $^{25} \mathrm{~A}$ meta-analysis of interventions performing pharmacist-led medication reconciliation alone at hospital transition found a relative risk reduction in all-cause readmissions of $19 \%$ by the intervention across included studies. ${ }^{16}$ This meta-analysis only included studies conducting medication reconciliation, which is only one facet of the planned intervention. It is expected that the intervention in the REMAIN HOME study will be more effective than medication reconciliation alone. A hospital discharge programme involving medication reconciliation and telephone follow-up showed an absolute risk reduction in readmissions (combined with ED visits) of $14 \%$ 
in the general medicine sample. ${ }^{36}$ In a pharmacist-led, home-based medication review after discharge, $45 \%$ of patients in the control group experienced a readmission compared with $28 \%$ in the intervention, resulting in an absolute risk reduction of $17 \% .^{37}$

This study is powered to detect a smaller effect size than the studies conducted by Jack $e t a l^{36}$ and Naunton and Peterson ${ }^{37}$ that are described above. This is a conservative estimate and smaller effect sizes are also likely to be clinically important. We expect only minimal variation in cluster sizes and so have not allowed for varying cluster sizes. With 14 clusters, and 20 patients recruited per cluster per month (except during the transition phase), it gives an expected total sample size of 2240 participants. Using these estimates and expected loss to follow-up of approximately $20 \%$, the design will have in the region of $80 \%-90 \%$ power to detect a change in proportions of unplanned readmissions from 0.3 to 0.2 for a range of ICCs (from 0.05 to 0.15 ).

\section{Randomisation}

The unit of randomisation is the study medical centre, not the participant. Once 14 medical centres have provided consent to be involved in the study, each enrolled medical centre will be randomised to a transition step by the study statistician $(\mathrm{KH})$. This will determine how long the medical centre is in the control and intervention phase. Stratified randomisation will be used to achieve an even balance of medical centres across the two geographical regions the study is set over while maintaining randomisation. Each medical centre will be informed of their randomisation status 1 month prior to their transition phase to be able to make the necessary arrangements for the pharmacist to integrate into the medical centre at the required time.

\section{Allocation concealment and blinding}

Research assistants recruiting participants will remain blinded to the randomisation schedule and will have no contact with the practice pharmacists throughout the study to ensure allocation concealment. Once the patient has been enrolled, the research assistant will notify the project coordinator who will pass the participant's details on to the practice pharmacist performing the intervention. If the medical centre the participant attends is in the control phase, the medical centre will not be notified.

Blinding of the intervention to staff at the medical centre and to participants is not possible as the presence of the pharmacist in the medical centre will indicate they are in the intervention phase. The statistician performing the analysis will be blinded to which group is the control and intervention group.

\section{Data collection}

Data will be collected from the participant's hospital and medical centre records by the project coordinator to determine whether a participant experienced a primary or secondary outcome.
Demographics and reason for admission at enrolment and subsequent admissions in the 12-month follow-up will be collected through participant hospital records.

Medical centre records will be used to identify whether a discharge treatment plan was received and the timeliness and number of GP visits during the 12-month follow-up period for each participant.

The practice pharmacist will record the details around each interaction with the participant in their practice medical notes as per normal standards. Pharmacist will also complete forms for each participant in the intervention phase to record their activities (see online supplementary file 1) at the initial consultation and follow-up(s). These records will be based on the DOCUMENT system that classifies drug-related problems and recommendations into coded categories and subcategories. ${ }^{38}$

Feedback on the acceptability, benefits and challenges of the GP-pharmacist model of care from the practice pharmacists, GPs, community pharmacies and participants (intervention only) will be collected (see online supplementary file 2 ). The views and experiences of the GPs will be measured through the validated questionnaire, Attitudes Toward Collaboration Instrument for GPs (ATCI-GP) ${ }^{28}$ Participation in these surveys will be optional.

\section{Data management and monitoring}

All data collected from participants, pharmacists and medical centre staff will remain confidential at all times. Paper documents will be stored securely in a locked cabinet. Electronic records will be stored as a database on a password-protected server. This is a high-quality, secure server and is backed up regularly.

The quality of collected data, recruitment and retention rates will be monitored throughout the trial.

This model of care is associated with minimal risk of harm. At all times during the trial, the participant will be under the care of their GP. GPs in the enrolled medical centres are not part of the research team and therefore will be an independent reviewer to assess and report any adverse events that occur during the study that may be related to the intervention. Any adverse events that come to the attention of the practice pharmacist or research team thought to be related to the intervention will be forwarded to the study safety committee. A study safety committee will be established that will consist of clinicians that are independent to the research project and will review any adverse events reported or thought to be related to the intervention.

\section{Analysis}

An intention-to-treat analysis will be conducted. All participants will be included in the analysis. Each medical centre will be classified as being in the intervention or the control phase based on their prespecified randomised cross-over time, regardless of whether crossover is achieved at that time. ${ }^{32}$ 
In the primary analysis, differences in readmissions will be modelled using a mixed effects Poisson regression model with an offset to incorporate the number of days of follow-up, a random effect for cluster and a fixed effect for each step to account for any temporal trend. Temporal trends may include seasonal variation in readmissions or changes in practice. We also intend to allow for both levels of clustering at the analysis stage-clustering by hospital and medical centre. This will be done by including both a random effect for medical centres and hospitals. If this model does not converge, we will include a fixed effect for hospital (as there will not be many hospitals) and a random effect for medical centre. In addition, a sensitivity analysis will be conducted with a random effect for pharmacist. This will allow for pharmacists working across multiple medical centres. Follow-up time will be the number of days from index admission discharge to the earliest of 12 months post discharge, death or loss to follow-up.

Secondary analysis will be conducted using similar techniques but using different link functions as appropriate. Descriptive statistics will be used to report the level of agreement with survey statements used to elicit the views of the intervention from GPs, practice pharmacists and participants involved in the study.

We will also report estimates of intracluster correlations.

Subgroup analyses will be hypothesis generating to assess whether the effect of the intervention varies by:

i. age: $<65$ years, $65-74$ years, $\geq 75$ years;

ii. Aboriginal community controlled health organisations versus non-aboriginal community controlled health organisations;

iii. level of patient frailty (defined by the Clinical Frailty Scale ${ }^{39}$ ): nine categories from 1 (very fit) to 9 (terminally ill);

iv. number of follow-up appointments with practice pharmacist;

v.time to consultation after discharge: $\leq 5$ days versus $>5$ days;

vi. Socio-Economic Indexes for Areas: quantiles from most disadvantaged to least disadvantaged;

vii. number and type of comorbidities at index hospital discharge;

viii.primary reason for indexed admission;

ix. number of prescribed medicines at index hospital discharge: $1-4,5-10$ and $>10$;

$\mathrm{x}$. residing in a residential aged care facility (RACF) versus non-RACF.

\section{Economic evaluation}

The direct healthcare costs that are associated with the intervention will be measured and compared with the costs associated with usual care. The intervention costs will include pharmacist time to deliver the collaborative service. The resource use for both intervention and control periods will also be estimated by recording the number, length and diagnosis-related group (DRG) allocations for unplanned hospital readmissions, the number and triage code for ED presentations and the number of GP visits, over the period of study follow-up. The primary source of hospital cost estimates, by DRG, will be derived from the National Efficient Cost and National Efficient Price data that are collected and published annually by Australia's Independent Hospital Pricing Authority (IHPA). ${ }^{40}$ This analysis will be restricted to direct medical and staffing costs to produce a meaningful basis of comparison with hospitals, primary carers, government agencies and private health insurers. The cost analysis will adjust for time preference using discounting and for clustering on location.

\section{Ethics and dissemination}

Ethics approval has been obtained through the Royal Brisbane and Women's Hospital Human Research Ethics Committee (HREC/16/QRBW/410).

The findings from this study will be disseminated through peer-reviewed publications, conferences and reports to key stakeholders. The results will help inform state, federal and private stakeholders operating in the health sector to implement evidence-based models for the transition of care of patients with chronic and complex diseases between hospital and the community settings. The evidence gained from this study will also inform the establishment of funding models to support the role of pharmacists in general practice medical centres in Australia by considering the clinical, humanistic and economic impacts.

\section{Author affiliations}

${ }^{1}$ School of Pharmacy, University of Queensland, Brisbane, Queensland, Australia ${ }^{2}$ Department of Public Health, Epidemiology and Biostatistics, University of Birmingham, Birmingham, UK

${ }^{3}$ Department of Internal Medicine and Clinical Epidemiology, Princess Alexandra Hospital, Brisbane, Queensland, Australia

${ }^{4}$ Southern School of Medicine, University of Queensland, Brisbane, Queensland, Australia

${ }^{5}$ Department of Pharmacy, Royal Brisbane and Women's Hospital, Metro North Hospital and Health Service, Brisbane, Queensland, Australia

${ }^{6}$ Brisbane South PHN, Brisbane, Australia

${ }^{7}$ School of Medicine, University of Queensland, Brisbane, Queensland, Australia

${ }^{8}$ Centre for the Business and Economics of Health, University of Queensland,

Brisbane, Queensland, Australia

${ }^{9}$ Poche Centre for Indigenous Health, University of Queensland, Brisbane,

Queensland, Australia

${ }^{10}$ Faculty of Medicine and Health Sciences, University of East Anglia Norwich

Medical School, Norwich, UK

${ }^{11}$ Department of Pharmacy, Monash Health, Clayton, Victoria, Australia

${ }^{12}$ Centre for Medicine Use and Safety, Monash University Faculty of Pharmacy and Pharmaceutical Sciences, Parkville, Victoria, Australia

${ }^{13}$ Mater Misericordiae Health Services Brisbane Ltd, South Brisbane, Queensland, Australia

${ }^{14}$ School of Primary Health Care, Monash University, Notting Hill, Victoria, Australia

Acknowledgements The authors would like to acknowledge the advice and input from the project reference group during the development of the study protocol.

Contributors $\mathrm{CF}$ and $\mathrm{HF}$ contributed equally to this work and led the development of this protocol. CF, HF, NC, IC, IW, IS, KH, LC, JW, CK, CN, GR and SK were all involved in developing the study protocol, design and funding proposal. KH provided the support for the development of the statistical analysis plan. JW and LC provided the support for the development of the economic analysis plan. HF, CF, NC, IC, IW, IS, KH, LC, JW and NS have been involved in the reviewing and editing the protocol manuscript. All authors have reviewed and approved the final version. 
Funding This work is supported by HCF Research Foundation, Brisbane North PHN and Brisbane South PHN.

Competing interests IW is the chair of Brisbane South PHN. This position is not dependent on the success of the project. Representatives of the HCF Research Foundation, Brisbane South PHN and Brisbane North PHN have been invited to be a part of the project reference group to provide input and advice on the study design. The project reference group plays no role in data analysis, interpretation of data or the decision to submit findings for publication.

Ethics approval Royal Brisbane \& Women's Hospital Human Research Ethics Committee.

Provenance and peer review Not commissioned; externally peer reviewed.

Open Access This is an Open Access article distributed in accordance with the Creative Commons Attribution Non Commercial (CC BY-NC 4.0) license, which permits others to distribute, remix, adapt, build upon this work non-commercially, and license their derivative works on different terms, provided the original work is properly cited and the use is non-commercial. See: http://creativecommons.org/ licenses/by-nc/4.0/

(c) Article author(s) (or their employer(s) unless otherwise stated in the text of the article) 2017. All rights reserved. No commercial use is permitted unless otherwise expressly granted.

\section{REFERENCES}

1. Australian Council on Safety and Quality in Health Care. Second national report on improving patient safety: improving medication safety. Canberra: Australian Council on Safety and Quality in Health Care, 2002

2. Howell S, Coory M, Martin J, et al. Using routine inpatient data to identify patients at risk of hospital readmission. BMC Health Serv Res 2009;9:1-9.

3. Jencks SF, Williams MV, Coleman EA. Rehospitalizations among patients in the Medicare fee-for-service program. N Engl J Med 2009;360:1418-28.

4. Davies EC, Green CF, Mottram DR, et al. Emergency re-admissions to hospital due to adverse drug reactions within 1 year of the index admission. Br J Clin Pharmacol 2010;70:749-55

5. Wimmer BC, Dent E, Bell JS, et al. Medication regimen complexity and unplanned hospital readmissions in older people. Ann Pharmacother 2014;48:1120-8.

6. Pal A, Babbott S, Wilkinson ST. Can the targeted use of a discharge pharmacist significantly decrease 30 -day readmissions? Hosp Pharm 2013;48:380-8

7. Picker D, Heard K, Bailey TC, et al. The number of discharge medications predicts thirty-day hospital readmission: a cohort study. BMC Health Serv Res 2015;15:1-8.

8. Bonnet-Zamponi D, d'Arailh L, Konrat C, et al; Optimization of Medication in AGEd study group. Drug-related readmissions to medical units of older adults discharged from acute geriatric units: results of the optimization of medication in AGEd multicenter randomized controlled trial. J Am Geriatr Soc 2013;61:113-21.

9. Unroe KT, Pfeiffenberger T, Riegelhaupt S, et al. Inpatient medication reconciliation at admission and discharge: a retrospective cohort study of age and other risk factors for medication discrepancies. Am $J$ Geriatr Pharmacother 2010;8:115-26.

10. Belleli E, Naccarella L, Pirotta M. Communication at the interface between hospitals and primary care - a general practice audit of hospital discharge summaries. Aust Fam Physician 2013;42:886-90.

11. Kilcup M, Schultz D, Carlson J, et al. Postdischarge pharmacist medication reconciliation: impact on readmission rates and financial savings. J Am Pharm Assoc 2013;53:78-84.

12. Coleman EA, Smith JD, Raha D, et al. Posthospital medication discrepancies: prevalence and contributing factors. Arch Intern Med 2005;165:1842-7.

13. Hansen LO, Young RS, Hinami K, et al. Interventions to reduce 30-day rehospitalization: a systematic review. Ann Intern Med 2011;155:520-8.

14. Leppin AL, Gionfriddo MR, Kessler M, et al. Preventing 30-day hospital readmissions: a systematic review and meta-analysis of randomized trials. JAMA Intern Med 2014;174:1095-107.

15. Stowasser DA, Collins DM, Stowasser M. A randomised controlled trial of medication liaison services - patient outcomes. J Pharm Pract Res 2002;32:133-40.
16. Mekonnen AB, McLachlan AJ, Brien JA. Effectiveness of pharmacistled medication reconciliation programmes on clinical outcomes at hospital transitions: a systematic review and meta-analysis. BMJ Open 2016:6:e010003

17. Kwan JL, Lo L, Sampson M, et al. Medication reconciliation during transitions of care as a patient safety strategy: a systematic review. Ann Intern Med 2013:158:397-403.

18. Vashi AA, Fox JP, Carr BG, et al. Use of hospital-based acute care among patients recently discharged from the hospital. JAMA 2013;309:364-71.

19. Mueller SK, Sponsler KC, Kripalani S, et al. Hospital-based medication reconciliation practices: a systematic review. Arch Intern Med 2012;172:1057-69.

20. Hawes EM, Maxwell WD, White SF, et al. Impact of an outpatient pharmacist intervention on medication discrepancies and health care resource utilization in posthospitalization care transitions. J Prim Care Community Health 2014;5:14-18.

21. Arnold ME, Buys L, Fullas F. Impact of pharmacist intervention in conjunction with outpatient physician follow-up visits after hospital discharge on readmission rate. Am J Health Syst Pharm 2015;72:S36-42.

22. Tedesco GW, McConaha JL, Skomo ML, et al. A pharmacist's impact on 30-day readmission rates when compared to the current standard of care within a patient-centered medical home: a pilot study. $J$ Pharm Pract 2016;29:368-73.

23. Holland R, Desborough J, Goodyer L, et al. Does pharmacist-led medication review help to reduce hospital admissions and deaths in older people? A systematic review and meta-analysis. Br J Clin Pharmacol 2008;65:303-16.

24. Polinski JM, Moore JM, Kyrychenko P, et al. An insurer's care transition program emphasizes medication reconciliation, reduces readmissions and costs. Health Aff 2016;35:1222-9.

25. Freeman C, Cottrell N, Rigby D, et al. The Australian practice pharmacist. J Pharm Pract Res 2014;44:240-8.

26. Freeman C, Rigby D, Aloizos J, et al. The practice pharmacist: a natural fit in the general practice team. Aust Prescr 2016;39:211-4

27. Tan EC, Stewart K, Elliott RA, et al. Pharmacist services provided in general practice clinics: a systematic review and meta-analysis. Res Social Adm Pharm 2014;10:608-22.

28. Van C, Costa D, Mitchell B, et al. Development and validation of a measure and a model of general practitioner attitudes toward collaboration with pharmacists. Res Social Adm Pharm 2013;9:688-99.

29. Hazen AC, Sloeserwij VM, Zwart DL, et al. Design of the POINT study: Pharmacotherapy Optimisation through Integration of a Nondispensing pharmacist in a primary care Team (POINT). BMC Fam Pract 2015;16:76.

30. Chan AW, Tetzlaff JM, Altman DG, et al. SPIRIT 2013 statement: defining standard protocol items for clinical trials. Ann Intern Med 2013;158:200-7.

31. Campbell MK, Piaggio G, Elbourne DR, et al; CONSORT Group. Consort 2010 statement: extension to cluster randomised trials. BMJ 2012;345:e5661.

32. Hemming K, Haines TP, Chilton PJ, et al. The stepped wedge cluster randomised trial: rationale, design, analysis, and reporting. $B M J$ 2015;350:h391.

33. Hussey MA, Hughes JP. Design and analysis of stepped wedge cluster randomized trials. Contemp Clin Trials 2007;28:182-91.

34. Hemming K, Lilford R, Girling AJ. Stepped-wedge cluster randomised controlled trials: a generic framework including paralle and multiple-level designs. Stat Med 2015;34:181-96.

35. Campbell MK, Fayers PM, Grimshaw JM. Determinants of the intracluster correlation coefficient in cluster randomized trials: the case of implementation research. Clin Trials 2005;2:99-107.

36. Jack BW, Chetty VK, Anthony D, et al. A reengineered hospital discharge program to decrease rehospitalization: a randomized trial. Ann Intern Med 2009:150:178-87.

37. Naunton M, Peterson GM. Evaluation of home-based follow-up of high-risk elderly patients discharged from hospital. J Pharm Pract Res 2003;33:176-82.

38. Williams M, Peterson GM, Tenni PC, et al. DOCUMENT: a system for classifying drug-related problems in community pharmacy. Int J Clin Pharm 2012;34:43-52.

39. Rockwood K, Song X, MacKnight C, et al. A global clinical measure of fitness and frailty in elderly people. CMAJ 2005;173:489-95.

40. Independent Hospital Pricing Authority. The pricing framework for Australian public hospital services 2016-17. Sydney, 2015.

41. Independent Hospital Pricing Authority. National efficient price determination 2016-17. Sydney, 2016. 